\title{
A DFT based ligand field study of the EPR spectra of Co(II) and $\mathrm{Cu}(\mathrm{II})$ porphyrins
}

\author{
Mihail Atanasov ${ }^{\text {a,c }}$, Claude A. Daul ${ }^{\mathrm{a}, *}$, Marie-Madeleine Rohmer ${ }^{\mathrm{b}}$, \\ Tamilmani Venkatachalam ${ }^{\text {a }}$ \\ a Département de Chimie de l'Université de Fribourg, Chemin du Musée 9, 1700 Fribourg, Switzerland \\ ${ }^{\mathrm{b}}$ Laboratoire de Chimie Quantique, UMR 7177 CNRS/ULP 4 Rue Blaise Pascal Strasbourg, France \\ ${ }^{\mathrm{c}}$ Institute of General and Inorganic Chemistry, Bulgarian Academy of Sciences, 1113 Sofia, Bulgaria
}

M.A., C.D. and M.M.R. dedicate this work to the memory of late Prof. A. Schweiger.

\begin{abstract}
Using a DFT based Ligand Field treatment (LFDFT) of the electronic structure of Co(II) and $\mathrm{Cu}(\mathrm{II})$ porphyrins (CoP and CuP) we analyse the origin of their EPR spectra. From a comparison between theoretical result on Co model clusters $(\mathrm{CoP}$ and $\mathrm{CoP}-\mathrm{ZnP}$ dimer $)$ we conclude that the $g$-tensor values are very sensitive to the axial coordination which stabilizes a ${ }^{2} \mathrm{~A}_{1}$ ground state in good agreement with experimental data. In contrast, DFT overestimates $\mathrm{Cu}$-ligand covalency, leading to large discrepancy with experiments, and hence the orbital contribution to the computed $g$-values is too small. Using a numerical adjustment of nuclear charge for $\mathrm{Cu}$, a good agreement between the computed and the experimental $g$-tensor values is observed. The influence of the DFT functional on the calculated $g$-tensor is also discussed.
\end{abstract}

\section{Introduction}

The electronic properties of porphyrin $(\mathrm{P})$ complexes have attracted considerable experimental and theoretical interest since the middle of the century, initially because of their important role in biological processes such as photosynthesis and respiration and more recently because of their potential technological applications [1].

Experimental and theoretical studies on porphyrins focussed on the origin and the intensities of the lowest ligand to ligand absorption bands, the $\mathrm{Q}$ and the $\mathrm{B}$ bands, which are successfully explained by Gouterman's fourorbital model [2] and more recently reconsidered within the TD-DFT method $[3,4]$.

The use of highly correlated ab initio calculations has been until now limited to free base porphyrin [5-7], magnesium porphyrin [8] and zinc porphyrin [9] complexes. Tran-

\footnotetext{
* Corresponding author. Fax: +41263009738.

E-mail address: claude.daul@unifr.ch (C.A. Daul).
}

sition metal complexes still represent a severe computational challenge.

Spectroscopic data on the electronic properties due to the multiplet structure originating from $\mathrm{d}^{\mathrm{n}}$ configuration of the TM metal is rather scarce, because $\mathrm{d}-\mathrm{d}$ electronic transitions are hidden by more intense ligand-ligand $\left(\pi \rightarrow \pi^{*}\right)$ band. In this respect Electronic Spin-Resonance (ESR) spectroscopy yields unique information about the nature of open-shell ground state and its dependence on the chemical environment. ESR spectra of Co(II) tetraphenyl porphyrine in matrices have been reported by Van Doorslaer and Schweiger [10] and by Brown and Hoffman for the analogous $\mathrm{Cu}(\mathrm{II})$ compound [11].

Interpretation of ESR spectra of TM complexes is usually based on Ligand Field Theory which allows to connect the $g$ - and $A$-tensors values with some limited number of model parameters extracted from high-resolution electronic spectra [12]. Recently, we developed a general DFT based LF computational scheme (LFDFT) which we first applied to interpret ESR spectra of $N, N^{\prime}$-ethylenebis 
(acetylacetoneiminato) $\mathrm{Co}(\mathrm{II})$ (Coacacen) [13]. In this communication we report our results for the $g$-tensor values of $\mathrm{CoP}$ and $\mathrm{CuP}$ using the LFDFT approach. We intend to show that axial coordination and chemical environment are crucial in reproducing a correct ground state for these compounds. It is well known that DFT intrinsically overestimates TM-ligand covalency which becomes increasingly pronounced from left to the right of the TM series $[14,15]$. In this respect $\mathrm{CuP}$ is rather interesting.

It has been shown by comparing a vast amount of experimental information with DFT calculations on $\mathrm{CuCl}_{4}^{2-}$ that hybrid functionals give a better agreement by introducing more ionicity than pure GGA functionals [14]. Alternatively, it has been proposed to use pure GGA functionals in combination with modified TM nuclear charge. Thus a reduction of nuclear charge by 0.8 for $\mathrm{Cu}$ (II) has lead to an excellent agreement with optical and ESR data for a wide range of $\mathrm{Cu}$-amine complexes [15].

In this study, we comment on the use of these approaches for $\mathrm{CoP}$ and $\mathrm{CuP}$ in connection with their ESR spectra.

\section{Method and computational details}

Density Functional Calculations have been carried out using Amsterdam Density Functional (ADF) program [16-21] with the local spin density (LDA) and generalized gradient approximation (GGA). The LDA was applied with the Vosko-Wilk-Nusair (VWN) [22] local spin-density potential and the GGA was applied by using PerdewWang91 (PW91) [23,24] exchange-correlation functional. Large Slater type orbital (STO) (triple- $\zeta$ ZORA) basis sets with one polarization function and the frozen core approximation have been used up to $3 p$ for metals and up to 1 s for carbon and nitrogen. We used throughout this work nonrelativistic exchange-correlation functionals, i.e., neglect magnetic effects and retardation in the electron interaction, which roughly corresponds to the use of the Dirac-Coulomb operator in $\mathrm{ab}$ initio calculations.

Geometries of $\mathrm{CoP}$ and $\mathrm{CuP}$ (Fig. 1) have been optimised in $\mathrm{D}_{4 \mathrm{~h}}$ symmetry. The multiplet structure has been calculated using our LFDFT procedure described in Ref $[25,26]$. In short, an Average-of-Configuration (AOC) DFT calculation is carried out to yield Kohn-Sham eigenvalues and eigenvectors. For the case of $\mathrm{Cu}(\mathrm{II})$ this immediately gives the $(5 \times 5)$ ligand field matrix. For CoP, a DFT calculation of the manifold of all 120 Slater determinants (SD) has been used to provide this $(5 \times 5)$ matrix and in addition the Racah parameters $B$ and $C$. To access the $g$ tensor values, the spin-orbit coupling constant and the orbital reduction factor have been deduced from spin-orbit coupling ZORA calculations. Details about the computational procedure and its implementation are given in [13].

The effect of environment for MP ( $\mathrm{M}=\mathrm{Co}(\mathrm{II})$ and $\mathrm{Cu}(\mathrm{II}))$ has been studied using MP-ZnP dimers. Such a choice is justified in view of the experimental data [10]. For the sake of simplicity we adopt a $\mathrm{C}_{4 \mathrm{v}}$ symmetry for the dimer

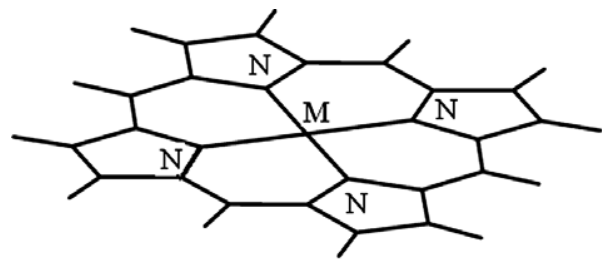

$\mathrm{M}=\mathrm{Co}(\mathrm{II}), \mathrm{Cu}$ (II)

Fig. 1. The $\mathrm{CuP}$ and $\mathrm{CoP}$ clusters adopted in the present study. LDA optimized $\mathrm{Co}-\mathrm{N}$ and $\mathrm{Cu}-\mathrm{N}$ bond distances are 1.955 and $2.001 \AA$, respectively. The $\mathrm{C}_{4}$ axis is parallel to $z$. The four $\mathrm{N}$ atoms are lying on the $x$ - and $y$-axes.

with a M-Zn separation of $3.4 \AA$ as reported from X-ray diffraction studies on analogous Ni(II) porphyrin [27].

The influence of the functional used in the DFT calculations was checked using the program package ORCA [28] (with a TZVP $+\mathrm{CP}(\mathrm{PPP}$ ) basis set for $\mathrm{Cu}$ and TZV for $\mathrm{N}$ and $\mathrm{C}$ ) which allows to compute ESR parameters using a hybrid B3LYP functional as well as other correlated methods $[29,30]$.

\section{Results and discussion}

\subsection{Ground state and EPR spectra of CoP}

Ligand field parameters, $B$ and $C$ values for $\mathrm{CoP}$ and $\mathrm{CoP}-\mathrm{ZnP}$ model clusters are reported in Table 1 . In $\mathrm{D}_{4 \mathrm{~h}}$ symmetry the five d-orbitals of $\mathrm{Co}(\mathrm{II})$ are split by antibonding $\sigma$ - and $\pi$-interactions with the porphyrin ligand. The orbital ordering and orbital shapes are displayed in Fig. 2. The splitting pattern of the orbitals can be easily rationalized within the angular overlap model (AOM).

$$
\begin{aligned}
& E\left(\mathrm{~b}_{1 \mathrm{~g}}\right)=3 e_{\sigma}^{\mathrm{eq}} \\
& E\left(e_{\mathrm{g}}\right)=2 e_{\pi}^{\mathrm{eq}} \\
& E\left(\mathrm{a}_{1 \mathrm{~g}}\right)=e_{\sigma}^{\mathrm{eq}}+2 e_{\sigma}^{\mathrm{ax}}-e_{4 \mathrm{~s}, 3 \mathrm{~d}} \\
& E\left(\mathrm{~b}_{2 \mathrm{~g}}\right)=0
\end{aligned}
$$

Table 1

LDFT data for $\mathrm{CoP}$ and $\mathrm{CoP}-\mathrm{ZnP}$

\begin{tabular}{lccl}
\hline & $\mathrm{CoP}^{\mathrm{a}}$ & CoP-ZnP & Exp. $^{\mathrm{c}}$ \\
\hline${ }^{2} \mathrm{~A}_{1}$ & 832 & 0 & - \\
${ }^{2} \mathrm{E}$ & 0 & 433 & - \\
${ }^{4} \mathrm{~B}_{2}$ & 5610 & 5211 & \\
${ }^{4} \mathrm{E}$ & 7970 & 8203 & \\
$B$ & 458 & 953 & \\
$C$ & 3645 & 2306 & 3.335 \\
$s(\mathrm{Co})$ & 400 & 400 & 3.335 \\
$g_{x}$ & 0.159 & 3.740 & 1.763 \\
$g_{y}$ & 0.159 & 3.740 & \\
$g_{z}$ & 4.327 & 1.617 &
\end{tabular}

LF-matrix elements (holes, $\mathrm{cm}^{-1}$ ):

\begin{tabular}{llllll} 
& $\left\langle x y\left|h^{\text {eff }}\right| x y\right\rangle$ & $\left\langle y z\left|h^{\text {eff }}\right| y z\right\rangle$ & $\left\langle z^{2}\left|h^{\text {eff }}\right| z^{2}\right\rangle$ & $\left\langle x z\left|h^{\text {eff }}\right| x z\right\rangle$ & $\left\langle x^{2}-y^{2}\left|h^{\text {eff }}\right| x^{2}-y^{2}\right\rangle$ \\
\hline a & 3032 & 1526 & 4250 & 1526 & -22713 \\
b & 7004 & 3484 & 6311 & 3484 & -19470 \\
c & Experimental data are given for the CoTPP doped into ZnTPP for the
\end{tabular}

(1c) site in Ref. [10]. 


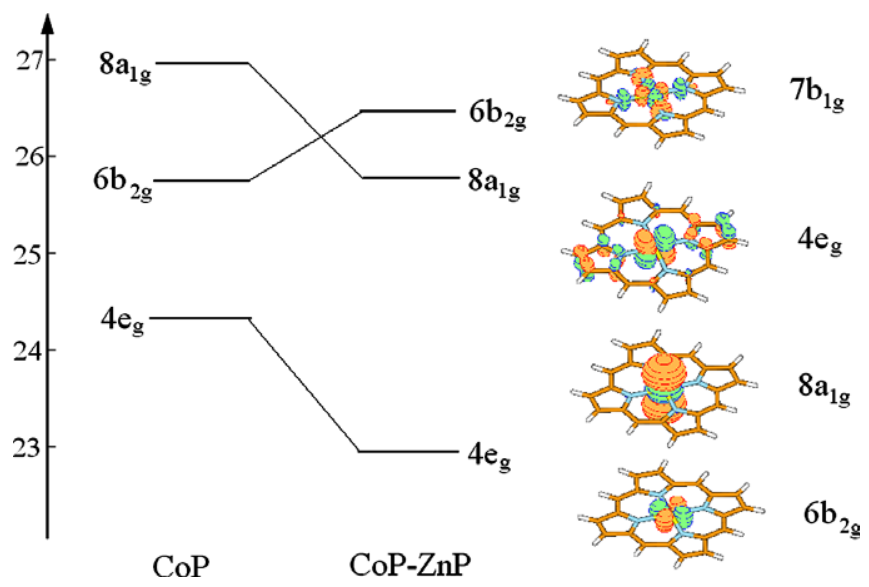

Fig. 2. Orbital energies (taken with a negative sign for holes) and orbital shapes for the MO's dominated by the Co(II) $3 \mathrm{~d}$ functions. Contour plots have been constructed using a value \pm 0.05 (electrons $/$ bohr $\left.^{3}\right)^{1 / 2}$. The LUMO $\left(7 b_{1 \mathrm{~g}}\right)$ has been drawn as well for the sake of completeness.

The highest $b_{1 g}\left(d_{x^{2}-y^{2}}\right)$ orbital is strongly destabilized through $\sigma$ overlap with the nitrogen ligators $\left(e_{\sigma}^{\mathrm{eq}}\right)$, while $e_{\mathrm{g}}\left(\mathrm{d}_{x z}, \mathrm{~d}_{y z}\right)$ undergoes a much weaker destabilisation via $\pi$ metal-ligand interaction $\left(e_{\pi}^{\mathrm{eq}}\right)$. In Eq. (1) $e_{\sigma}^{\mathrm{eq}}$ and $e_{\pi}^{\mathrm{eq}}$ are AOM parameters for the equatorial nitrogen ligands refering to a standard orientation $[31,32]$. Since there is no $\pi$ overlap within porphyrine plane the $\mathrm{b}_{2 \mathrm{~g}}\left(\mathrm{~d}_{x y}\right)$ orbital has a non-bonding character. The relative position of the $a_{1 g}\left(d_{z^{2}}\right)$ orbital is subject to two interactions with the porphyrine ring. First of all, it is shifted by the antibonding interaction arising from $\sigma$-overlap with the nitrogen orbitals of porhyrine. In addition there is strong stabilizing effect due to mixing between $3 \mathrm{~d}_{z^{2}}$ and $4 \mathrm{~s}$ orbitals of $\mathrm{Cu}(\mathrm{II})$ (accounted for by the term $-\mathrm{e}_{4 \mathrm{~s}, 3 \mathrm{~d}}$ in Eq. (1)) which belongs to the same irreducible representation $\left(\mathrm{a}_{1 \mathrm{~g}}\right)$ in $\mathrm{D}_{4 \mathrm{~h}}$ symmetry. The two effects almost compensate.

In the case of axial coordination the $\mathrm{d}_{z^{2}}$-orbital becomes strongly destabilized by $\sigma$-antibonding interaction (accounted for by an additional parameter $e_{\sigma}^{\mathrm{ax}}$ in Eq. (1)) which is significant in the $\mathrm{CoP}-\mathrm{ZnP}$ model cluster at a Co-Zn distance of $3.4 \AA$ (Fig. 2) and is of crucial importance in the determination of the nature of the ground state. This gives rise to two possibilities for the doublet ground state of CoP, i.e. ${ }^{2} \mathrm{E}_{\mathrm{g}}$ and ${ }^{2} \mathrm{~A}_{1 \mathrm{~g}}$ (Fig. 3a). The energy separation between these states is given, to 1st order, by

$$
\begin{aligned}
\Delta & =E\left({ }^{2} \mathrm{~A}_{1 \mathrm{~g}}\right)-E\left({ }^{2} \mathrm{E}_{\mathrm{g}}\right)=-E\left(\mathrm{a}_{1 \mathrm{~g}}\right)+E\left(e_{\mathrm{g}}\right)-5 B \\
& =-e_{\sigma}^{\mathrm{eq}}-e_{\sigma}^{\mathrm{ax}}(\mathrm{eff})+2 e_{\pi}^{\mathrm{eq}}-5 B
\end{aligned}
$$

where an effective parameter for axial ligation has been introduced:

$e_{\sigma}^{\mathrm{ax}}(\mathrm{eff})=2 e_{\sigma}^{\mathrm{ax}}-e_{4 \mathrm{~s}, 3 \mathrm{~d}}$

From Eqs. (2) and (3) it follows, that interelectronic repulsion and axial ligation favour a ${ }^{2} \mathrm{~A}_{1 \mathrm{~g}}$ ground state, while s-d mixing counteracts this interaction, leading to a ${ }^{2} \mathrm{E}_{\mathrm{g}}$ ground state in the case of CoP (Table 1).

In $\mathrm{D}_{4 \mathrm{~h}}$ symmetry the cubic ${ }^{4} \mathrm{~T}_{2 \mathrm{~g}}$ state splits into ${ }^{4} \mathrm{~B}_{2 \mathrm{~g}}$ and ${ }^{4} \mathrm{E}_{\mathrm{g}}$ states which are, respectively at about 5600 and
$8000 \mathrm{~cm}^{-1}$ above the ground state (cf. Table 1). Therefore, in a first approximation, the ground state manifold can be described by a three state model given in Eq. (4). In this equation we have used notations for irreducible representations of the $\mathrm{D}_{4}^{*}$ double group.

\begin{tabular}{l|lll}
$\left\langle\Psi|\hat{H}| \Psi^{\prime}\right\rangle$ & $\left|\Gamma_{7}\left({ }^{2} E\right)\right\rangle$ & $\left|\Gamma_{6}\left({ }^{2} A_{1}\right)\right\rangle$ & $\left|\Gamma_{6}\left({ }^{2} E\right)\right\rangle$ \\
\hline$\left\langle\Gamma_{7}\left({ }^{2} E\right)\right|$ & $\zeta / 2$ & 0 & 0 \\
$\left\langle\Gamma_{6}\left({ }^{2} A_{1}\right)\right|$ & 0 & $\Delta$ & $i \zeta \sqrt{3 / 2}$ \\
$\left\langle\Gamma_{6}\left({ }^{2} E\right)\right|$ & 0 & $-i \zeta \sqrt{3 / 2}$ & $-\zeta / 2$
\end{tabular}

This matrix $\mathbf{H}$ can easily be diagonalised using the following unitary transformation:

$\mathbf{E}=\mathbf{U}^{\dagger} \mathbf{H U}$

where

$\mathbf{U}=\left[\begin{array}{lll}1 & 0 & 0 \\ 0 & \cos \theta & -i \sin \theta \\ 0 & -i \sin \theta & \cos \theta\end{array}\right]$ and $\tan (2 \theta)=\frac{2 \zeta \sqrt{3 / 2}}{\Delta+\zeta / 2}$

The components of the $g$-tensor in the experimental spin hamiltonian $\beta \vec{B} \cdot \mathbf{g} \cdot \vec{S}$ are then easily obtained by calculating the corresponding components of the Zeeman hamiltonian: $\left\langle n \Gamma_{i} \pm|\vec{B} \cdot(\vec{l}+2 \vec{s})| n \Gamma_{i} \pm\right\rangle$. The result for the three relevant Kramers doublet reads:

$$
\begin{aligned}
\left|1 \Gamma_{7}\right\rangle: & g_{x x}=g_{y y}=g_{\perp}=0 ; \quad g_{z z}=g_{\|}=-4 \\
\left|1 \Gamma_{6}\right\rangle: & g_{x x}=g_{y y}=g_{\perp}=2 \cos ^{2} \theta+4 \sqrt{1.5} \sin \theta \cos \theta ; \\
g_{z z} & =g_{\|}=2 \cos ^{2} \theta \\
\left|2 \Gamma_{6}\right\rangle & : \quad g_{x x}=g_{y y}=g_{\perp}=2 \sin ^{2} \theta-4 \sqrt{1.5} \sin \theta \cos \theta ; \\
g_{z z} & =g_{\|}=2 \sin ^{2} \theta
\end{aligned}
$$

This three-state model explains nicely the main features of the $g$-tensor of CoP. Results obtained with this model are displayed in Fig. 3a,b. However, when comparing results with experiment, the effect of the whole manifold of quartet and doublet states on the ground state splitting has to be taken into account (Table 1).

The effect of spin-orbit coupling, $\zeta$ being the spin-orbit coupling constant, on the relative energies of the ${ }^{2} E_{g}$ and ${ }^{2} \mathrm{~A}_{1}$ states is illustrated in Fig. 3a, where energies are plotted as function of $e_{\sigma}^{\text {ax }}($ eff $)$. Inspection of Fig. 3 reveals that the ground state changes from ${ }^{2} \mathrm{E}_{\mathrm{g}}$ to ${ }^{2} \mathrm{~A}_{1 \mathrm{~g}}$ (Fig. 3a) when $e_{\sigma}^{a x}($ eff $)$ increases. Spin-orbit coupling splits the ${ }^{2} \mathrm{E}_{\mathrm{g}}$ state into a $\Gamma_{6}$ and a $\Gamma_{7}$ Kramers doublets and allows for a mixing between $\Gamma_{6}\left({ }^{2} \mathrm{E}_{\mathrm{g}}\right)$ and $\Gamma_{6}\left({ }^{2} \mathrm{~A}_{1 \mathrm{~g}}\right)$. Due to the interaction of these two states, an avoided crossing takes place. This change between the two ground states is of crucial importance for the $g$-tensor, as is seen from Fig. 3c. A large anisotropy of the $g$-tensor is observed when $\Gamma_{7}$ is ground state (spin-orbit component of ${ }^{2} \mathrm{E}_{\mathrm{g}}$ that does not mix with ${ }^{2} \mathrm{~A}_{1 \mathrm{~g}}$ ), where $g_{x, y}$ is almost zero and with a large $g_{z}$ value of 4.327. In this case, almost no dependence on $e_{\sigma}^{z}$ (eff) is observed. Going from a $\Gamma_{7}$ to a $\Gamma_{6}$ ground state, there is 

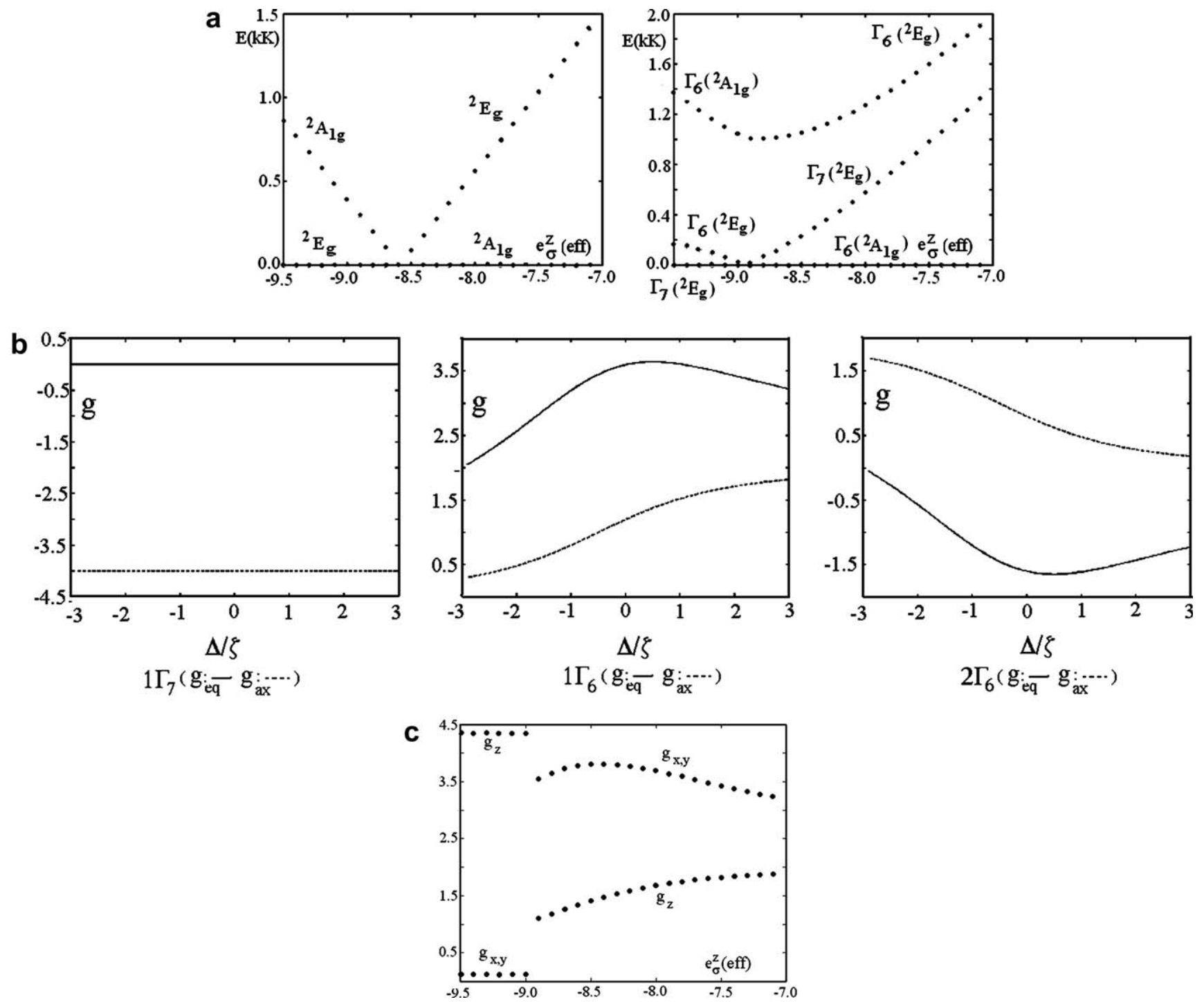

Fig. 3. (a) Term energies of the lowest ${ }^{2} \mathrm{~A}_{1 \mathrm{~g}}$ and ${ }^{2} \mathrm{E}_{\mathrm{g}}$ states of $\mathrm{Co}(\mathrm{II})$ in $\mathrm{CoP}-\mathrm{ZnP}$ without spin-orbit coupling (left) and taking spin-orbit coupling into account (right, $\zeta=-400 \mathrm{~cm}^{-1}, \mathrm{D}_{4}^{*}$ double group notations). (b) Components of the $g$-tensor calculated according to Eq. (6) for all three Kramers doublets. (c) $g$-tensor values obtained with the LFDFT model varying artificially the energy of the $\mathrm{a}_{1 \mathrm{~g}}\left(\mathrm{~d}_{z^{2}}\right)$-orbital; $\zeta=-400 \mathrm{~cm}^{-1}$; Ligand field matrix elements and $B$ and $C$ values are taken for $\mathrm{CoP}-\mathrm{ZnP}$ (see Table 1).

an abrupt change in the $g$-tensor values. That is, a large equatorial $g_{x, y}$ components and a $g_{z}$ value smaller than two result. This corresponds to the experimentally observed $g$-tensor components [10]. Comparing the results of Table 1, we can state that it is the extended $\mathrm{CoP}-\mathrm{ZnP}$ cluster which accounts for $g$-tensor anisotropy, allowing one to unambigously identify the nature of the ground state of $\mathrm{CoP}$ as dominated by the energy separation between the ${ }^{2} \mathrm{~A}_{1 \mathrm{~g}}$ and the ${ }^{2} \mathrm{E}_{\mathrm{g}}$ states. This also confirms the important role of the weak axial coordination in $\mathrm{CoP}-\mathrm{ZnP}$ already discussed on the basis of the experimental data [10].

\subsection{Ground state and EPR spectra of CuP}

$\mathrm{CuP}$ posseses a ${ }^{2} \mathrm{~B}_{1 \mathrm{~g}}$ ground state with a single electron in the $\mathrm{d}_{x^{2}-y^{2}} \sigma$ antibonding orbital. A MO diagram for orbitals occupied with holes is shown in Fig. 4, where the energy of $\mathrm{d}_{x^{2}-y^{2}}$ is taken as reference energy. As can be seen, the Kohn-Sham $\pi$ orbital $4 \mathrm{e}_{\mathrm{g}}$ is mainly of ligand character, the corresponding $\mathrm{MO}$ dominated by $\mathrm{d}-\mathrm{AO}$ being much lower in energy. This is a typical result for $\mathrm{Cu}$ (II) complexes. It is well known, from ESR and ENDOR experiments on $\mathrm{Cu}(\mathrm{II})$ complexes with nitrogen ligators, that the relative percentage of $\mathrm{Cu} 3 \mathrm{~d}$ character in $\sigma$-antibonding $\mathrm{d}_{x^{2}-y^{2}}$ is around $65 \%$ to $70 \%[33,34]$. In contrast, for $\mathrm{CuP}$ this amount is only $49 \%$. As for CoP, the $\mathrm{d}_{x y}\left(6 \mathrm{~b}_{2 \mathrm{~g}}\right)$ and $\mathrm{d}_{z^{2}}\left(8 \mathrm{a}_{1 \mathrm{~g}}\right)$ orbitals are less affected by this effect remaining mainly pure d-orbitals. EPR parameters computed from these Kohn-Sham orbitals are listed in Table 2. At variance with experimental data, the computed results show a much too small orbital contribution to the $g$-tensor values, related also to a rather small value of the spin-orbit coupling constant. One way to increase the orbital contribu- 


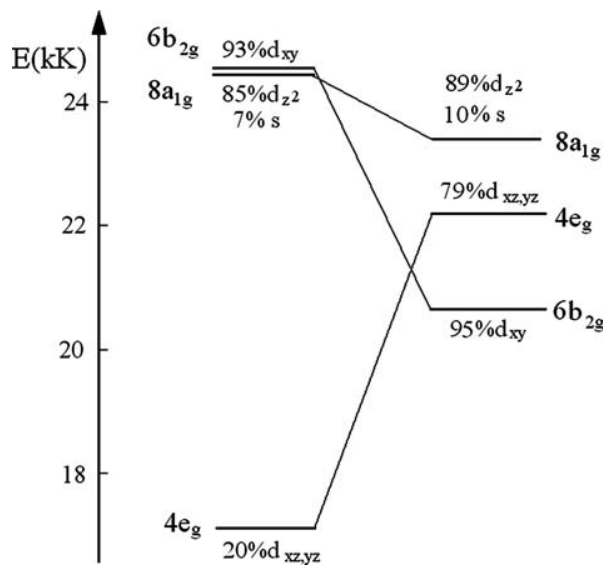

Fig. 4. Orbital energies (taken with a negative sign for holes) and compositions for $\mathrm{CuP}$. Adopted values for the nuclear charge $q$ of $\mathrm{Cu}(\mathrm{II})$ are 29 (left) and 28.2 (right).

tions to the $g$-tensor is to impose a relocalization of the metal d-orbitals. To this end we adopted a recipe proposed by Deeth for $\mathrm{Cu}(\mathrm{II})$ amine complexes [15], which consists in taking a value of 28.2 for the nuclear charge of $\mathrm{Cu}(\mathrm{II})$ in order to reduce the metal-ligand covalency. The corresponding results are included in Fig. 4 and Table 2. One can see, a dramatic change in orbital energies, i.e. lowering of $6 \mathrm{~b}_{2 \mathrm{~g}}$ and $8 \mathrm{a}_{1 \mathrm{~g}}$ and increase of $4 \mathrm{e}_{\mathrm{g}}$ and more importantly, increase of d-orbital percentages in $4 \mathrm{e}_{\mathrm{g}}$ and $6 \mathrm{~b}_{1 \mathrm{~g}}$ orbitals, leading to an orbital reordering. This also affects the spin-orbit coupling constant and the computed $g$-tensor values, which are now in reasonable agreement with experimental ones (Table 2). As expected, axial coordination does not affect neither the orbital sequence nor the computed $g$-tensor.

\subsection{Influence of the functional on the EPR results}

In Table 3 we report calculated $g$-tensor parameters for $\mathrm{CoP}$ and $\mathrm{CuP}$ with the hybrid B3LYP functional. For $\mathrm{CuP}$ there is an excellent agreement between calculated and experimental value for $g_{x, y}$. However, the $g_{z}$ value is off by $30 \%$, as previously reported for $\mathrm{Cu}(\mathrm{II})$ bispidine complexes [35]. A somewhat better agreement is obtained from LFDFT calculation (see Table 2) where 3d-metal electrons
Table 3

$g$-values of $\mathrm{CuP}$ and $\mathrm{CoP}$ calculated using the B3LYP functional

\begin{tabular}{llllll}
\hline & CuP & & & CoP & \\
\cline { 2 - 3 } \cline { 5 - 6 } & B3LYP & Exp. $^{\mathrm{a}}$ & & B3LYP & Exp. $^{\mathrm{b}}$ \\
\hline$g_{x, y}$ & 2.047 & 2.045 & & 2.369 & 3.335 \\
$g_{z}$ & 2.135 & 2.190 & & 2.005 & 1.763 \\
\hline
\end{tabular}

${ }^{\mathrm{a}}$ Ref. [11].

b Experimental data are given for the CoTPP doped into ZnTPP [(1c) species in Ref.: [10].

have been localized in a special treatment as discussed in the previous section.

The agreement between computed and experimental values for CoP is less satisfactory. There is a correct description of the ${ }^{2} \mathrm{~A}_{1 \mathrm{~g}}\left(\mathrm{~d}_{x^{2}-y^{2}}^{2} \mathrm{~d}_{z^{2}}^{1}\right)$ ground state of CoP with $g_{x, y}$ larger than 2 and $g_{z}$ close to 2.00 (orbital free component). However, d-orbital contributions to spin-only $g$-tensor are too low. The reason for this might be twofold. First, contribution from axial coordination could not be taken into account in the B3LYP calculation. Second, the perturbational approach which is justified by the larger energy gap between $\mathrm{d}_{x^{2}-y^{2}}$ and the $\mathrm{d}_{x y}, \mathrm{~d}_{x z, y z}$ levels in the case of $\mathrm{CuP}$, is certainly too limited for $\mathrm{CoP}$ with a nearly degenerate ${ }^{2} A_{1 g}-{ }^{2} E_{g}$ manifold of states (see Section 3.1).

\section{Conclusion}

In this Letter we have applied the LFDFT approach to compute and interpret ESR data for $\mathrm{CoP}$ and $\mathrm{CuP}$ complexes. On this basis we have unambiguously assigned the ground state of $\mathrm{CoP}$ as a mixture of Kramers doublet from ${ }^{2} \mathrm{E}_{\mathrm{g}}$ and ${ }^{2} \mathrm{~A}_{1 \mathrm{~g}}$ states with a clear dominance of latter. We show, that the influence of axial coordination should be taken into account in order to reproduce $g$-tensor values in the case of $\mathrm{Co}$ (II) doped into $\mathrm{ZnP}$. In this case the conventional DFT programs like ADF or ORCA that are based on perturbation theory do not predict correctly the $g$-tensor since the spin-orbit coupling constant has the same order of magnitude as the ${ }^{2} \mathrm{E}-{ }^{2} \mathrm{~A}_{1}$ energy separation. However, the LFDFT model we used in the present work does predict the $g$-tensor correctly. The small deviation between the predicted and the observed value in Table 1 is due to the influence of the next-nearest neighbours in the bulk of the crystal. Axial coordination of a variable

Table 2

LDFT data for $\mathrm{CuP}$ and $\mathrm{CuP}-\mathrm{ZnP}^{\mathrm{a}}$

\begin{tabular}{|c|c|c|c|c|c|c|c|}
\hline \multirow[t]{3}{*}{ Electronic transition } & \multicolumn{2}{|l|}{$\mathrm{CuP}$} & \multicolumn{2}{|l|}{$\mathrm{CuP}$} & \multicolumn{2}{|c|}{$\mathrm{CuP}-\mathrm{ZnP}$} & \multirow[t]{3}{*}{ Exp. $^{\text {b }}$} \\
\hline & \multicolumn{2}{|l|}{$q=29$} & \multicolumn{2}{|c|}{$q=28.2$} & \multicolumn{2}{|c|}{$q=28.2$} & \\
\hline & $\varsigma=0.0$ & $\varsigma=450.0$ & $\varsigma=0.0$ & $\varsigma=600$ & $\varsigma=0.0$ & $\varsigma=578$ & \\
\hline$b_{2 g} \rightarrow b_{1 g}$ & 24155 & 24191 & 20670 & 20621 & 20816 & 20772 & - \\
\hline \multirow{2}{*}{$e_{\mathrm{g}} \rightarrow \mathrm{b}_{1 \mathrm{~g}}$} & 16235 & 15985 & 22106 & 21459 & 22324 & 21811 & - \\
\hline & & 16469 & & 22535 & & 22729 & \\
\hline
\end{tabular}


strength can also explain the large changes of the $g$-tensor values found in continuous wave and pulse electron paramagnetic resonance measurements in different $\mathrm{Co}(\mathrm{II})$ porphyrines [10].

We find that in the case of $\mathrm{CuP}$, the $g$-tensor values are not properly reproduced by the LFDFT method, which underestimates orbital contributions. The situation can be improved by using localized orbitals obtained via a modification of the $\mathrm{Cu}(\mathrm{II})$ nuclear charge. A similar effect can be achieved by increasing the ionic character of the $\mathrm{Cu}-$ nitrogen bond as is implicitly done by the use of hybrid B3LYP functional.

More work should be devoted towards a non-empirical description of late transition metal complexes.

\section{Acknowledgement}

This work was supported by the Swiss National Science Foundation and by the State Secretariat for Science and Education. C.A.D. thanks the Université Louis Pasteur de Strasbourg for an invited professorship. The authors also thank Dr. F. Neese for supplying ORCA as well as for valuable suggestions.

\section{References}

[1] K.M. Kadish, R. Guilard, K.M. Smith (Eds.), The Porphyrin Handbook, Academic Press, 1999.

[2] M. Gouterman, J. Mol. Spectrosc. 6 (1961) 138.

[3] E.J. Baerends, G. Ricciardi, A. Rosa, S.J.A. van Gisbergen, Coord. Chem. Rev. 230 (2002) 5.

[4] G. Ricciardi, A. Rosa, E.J. Baerends, S.J.A. van Gisbergen, J. Am. Chem. Soc. 124 (2002) 12319

[5] M. Merchán, E. Ortí, B.O. Roos, Chem. Phys. Lett. 226 (27) (1994).

[6] S. Yamamoto, H. Tatewaki, O. Kitao, G.H.F. Diercksen, Theor. Chem. Acc. 106 (2001) 287.

[7] Y. Yamamoto, T. Noro, K. Ohno, Int. J. Quantum Chem. 42 (1992) 1563.

[8] J. Šeda, J.V. Burda, J. Leszczynski, J. Comp. Chem. 26 (2005) 294.

[9] J.G. Goll, K.T. Moore, A. Ghosh, M.J. Therien, J. Am. Chem. Soc. 118 (1996) 8344.
[10] S. Van Doorslaer, A. Schweiger, Phys. Chem. Chem. Phys. 3 (2001) 159 .

[11] T.G. Brown, B.M. Hoffman, Mol. Phys. 39 (1980) 1073.

[12] M. Hitchman, B. Figgis, Introduction to Ligand Field Theory, Willey, VCH, Weinheim, 2000.

[13] M. Atanasov, E.J. Baerends, P. Baettig, R. Bruyndonckx, C. Daul, C. Rauzy, M. Zbiri, Chem. Phys. Lett. 399 (4-6) (2004) 433.

[14] R.K. Szilagyi, M. Metz, E.I. Solomon, J. Phys. Chem. A 106 (2002) 2994.

[15] R.J. Deeth, J. Chem. Soc., Dalton Trans. (2001) 664.

[16] A. Bérces et al., ADF2004.01; SCM, Theoretical Chemistry, Vrije Universiteit, Amsterdam, The Netherlands, 2004. Available from: $<$ http://www.scm.com $>$.

[17] E.J. Baerends, D.E. Ellis, P. Ros, Chem. Phys. 2 (1993) 41.

[18] P.M. Boerrigter, G. te Velde, E.J. Baerends, Int. J. Quantum Chem. 33 (1988) 87

[19] G. teVelde, E. Baerends, J. Comput. Phys. 99 (1992) 84.

[20] G. te Velde, F.M. Bickelhaupt, E.J. Baerends, C. Fonesca Guerra, S.J.A. van Gisbergen, J.G. Snijders, T. Ziegler, J. Comput. Chem. 22 (2001) 931.

[21] E. van Lenthe, E.J. Baerends, J.G. Snijders, J. Chem. Phys. 99 (1993) 4597.

[22] S.H. Vosko, L. Wilk, M. Nusair, Can. J. Phys. 58 (1980) 1200.

[23] J.P. Perdew, Y. Wang, Phys. Rev. B 33 (1986) 8800.

[24] J.P. Perdew, J.A. Chevary, S.H. Vosko, K.A. Jackson, M.R. Pederson, D.J. Singh, C. Fiolhais, Phys. Rev. B 46 (1992) 6671; Error correction: Phys. Rev. B 48 (1993) 4978.

[25] M. Atanasov, C.A. Daul, C. Rauzy, Chem. Phys. Lett. 367 (2003) 737.

[26] M. Atanasov, C.A. Daul, C. Rauzy, Structure \& Bonding 106 (2004) 97.

[27] W. Jentzen, I. Turowska-Tyrk, W.R. Scheidt, J.A. Shelnutt, Inorg. Chem. 35 (1996) 3559.

[28] F. Neese, ORCA - An Ab Initio, DFT and Semiemprical SCF-MOPackage, Version 2.4-41, October 2005.

[29] F. Neese, J. Chem. Phys. 122 (2005) 034107/1

[30] F. Neese, J. Chem. Phys. 118 (2003) 3939.

[31] C.K. Jørgensen, R. Papalardo, H.-H. Schmidtke, J. Chem. Phys. 39 (1963) 1422.

[32] C.E. Schäffer, C.K. Jørgensen, Mol. Phys. 9 (1965) 401

[33] A.G. Brenosa, M. Moreno, J.A. Aramburu, J. Phys.: Condens. Matter 3 (1991) 7743.

[34] M.Q. Ehsan, Y. Ohba, S. Yamauchi, M. Iwaizumi, Chem. Lett. 151 (1995).

[35] M. Atanasov, P. Comba, B. Martin, V. Müller, G. Rajaraman, H. Rohwer, S. Wunderlich, J. Comput. Chem. 27 (2006) 1263. 